\title{
Maintaining Privacy on a Line
}

\author{
Evangelos Kranakis Danny Krizanc
}

January 22, 2011

\begin{abstract}
Consider a situation where people have to choose among a sequence of $n$ linearly ordered positions to perform some task requiring a certain amount of privacy. Which position should one choose so as to maximize one's privacy, i.e., minimize the chances that one of your neighboring positions becomes occupied by a later arrival? In this paper, we attempt to answer this question under a variety of models for the behavior of the later arrivals. Our results suggest that for the most part one should probably choose one of the extreme positions (with some interesting exceptions). We also suggest a number of variations on the problem that lead to many open problems.
\end{abstract}

\section{Introduction}

The question of digital privacy and how to protect it has a long history of study in computer science and it raises a number of interesting algorithmic (as well as other) research problems [5]. But it is also the case that algorithmic issues may arise when considering physical privacy. Consider a situation where multiple public locations are available to perform an activity for which one prefers not to be observed closely or interrupted, i.e., one desires a certain amount of privacy. Examples might include choosing a table at a restaurant for an intimate dinner, choosing a seat in a theatre avoiding talkative patrons or choosing an ATM without any onlookers. Which location should one choose so as to maximize your privacy? If the situation were static and no other parties were expected to arrive then this reduces to finding the location furthest from all currently occupied locations. But in the cases above, one expects that others will arrive later to occupy other locations. The question we consider here is how this might effect your choice.

A person walks into a bank with ATMs arranged on a line adjacent to each other. Upon arrival some of the ATMs are occupied and others are empty. Under these conditions which ATM should one pick so as to maximize her chances of maintaining privacy, i.e., minimize the chance that someone will occupy a position beside her? One's intuition might suggest that choosing one of the end positions is the best choice. It turns out the answer depends on many things but 
in particular on how one models the behavior of the persons who enter later. ${ }^{1}$

Our problem of maintaining privacy on a line may be modelled as follows. Consider a line consisting of $n$ linearly ordered postions, $1,2, \ldots, n$. Some of the positions are occupied and others are not. A position $i$ is called private if positions $i-1$ and $i+1$ are unoccupied (if they exist). Assume that the remaining unoccupied positions are to be filled in an order determined by a (possibly randomized) strategy that is known. For the given configuration of occupied and unoccupied positions with at least one private position, which private position should be chosen so as to maximize the (expected) number of positions filled before the strategy fills a neighboring position thus violating the privacy of the chosen position.

In this paper, we consider a variety of filling strategies and attempt to develop algorithms for maintaining privacy under each. The results of this study suggest that for the most part one's intuition is correct (the ends are best) with some interesting exceptions. We also examine a number of variations on the problem that might lead to a better understanding of physical privacy concerns and which suggest many interesting open questions.

\section{Related Work}

If one is interested in finding the most private position to place an object in the sense of it being furthest from all other occupied positions in some space this can be formulated as a version of the well-studied Obnoxious Facility Location Problem. (See [8] for a survey of results in this area.) But in our case this problem is trivially solved by taking the position at the mid-point of the largest gap of positions (i.e., contiguous sequence of empty positions) and it does not really answer our question. The question of privacy in querying sensors in a sensor network also shares some aspects of our question but again the concerns turn out to be different [10].

Most closely related to our question (at least for the case where the persons behave randomly) seems to be the following "unfriendly seating arrangement" problem posed by Freedman and Shepp [2]: There are $n$ seats in a row at a luncheonette and people sit down one at a time at random. They are unfriendly and so never sit next to one another (no moving over). What is the expected number of persons to sit down? Solutions to this problem were provided by Friedman, Rothman and MacKenzie [3, 7] who show that as $n$ tends to infinity the expected fraction of the seats that are occupied goes to $\frac{1}{2}-\frac{1}{2 e^{2}}$. (For a nice exposition of this and related problems see [1].) Georgiou et al. [4] consider the following generalization of this problem (the unfriendly theatre seating arrangement problem): People arrive one at a time to a theatre consisting of $m$ rows of length $n$. Being unfriendly they choose seats at random so that no one is in front of them, behind them or to either side. What is the expected number of

\footnotetext{
${ }^{1}$ Following this formulation of the problem we will refer to the people occupying positions on the line as persons though our results can be applied in any situation where the positions are linearly ordered and privacy is a concern.
} 
people in the theatre when it becomes full, i.e., it can not accommodate any more unfriendly people? They give bounds on the fraction of the theatre that is full (in the limit) for all $m$ and show that for $m=2$ this limit is $\frac{1}{2}-\frac{1}{4 e}$.

\section{The Privacy Problem on a Line}

For our initial study we assume that line is initially empty, i.e., all of the positions are unoccupied and one must choose the first position to occupy. (We will discuss the case where some positions are already occupied below.) Further we assume that in any natural filling strategy all private positions will be filled before any position violating a position's privacy will be chosen. If no private positions remain then we assume the random strategy for choosing a position where one chooses uniformly at random among all unoccupied positions. We note that other models of how the remaining positions are filled are possible and make some remarks on some of them below. Finally, we assume that once occupied a position will remain occupied until all positions are filled, i.e., nobody leaves once they enter.

For each of the scenarios we consider, we are interested in maximizing the expected number of filled positions before the privacy of the first chosen position will be violated, i.e., one of its neighboring positions is filled. We call a set of the occupied positions a configuration. We call a configuration saturated if the next position to be filled is forced to violate at least one position's privacy. It is fairly clear that, under the assumptions above, the time until the first position's privacy is violated can be divided into two phases: the time until saturation is reached and time until the random filling strategy violates its privacy once the configuration is saturated. As such we divide our analysis for each of the cases below into two parts: the time until saturation and the time until privacy violation once saturation has been achieved.

\subsection{Lazy behavior}

In this model one chooses the lowest number unoccupied position that provides privacy. (I.e., following the ATM room analogy we assume that positions are ordered according to their distance from the door and that persons, being naturally lazy, will always choose the first empty private spot.) The analysis of the first phase divides into two cases depending upon whether $n$ is even or odd.

If $n$ is even and you choose an odd numbered position then clearly the saturated configuration will consist of all of the odd positions and will contain $n / 2$ occupied positions. If you choose an even numbered position, $2 k$, then the saturated configuration consists of the $k-1$ odd positions before you (excluding $2 k-1$ ) and the $n / 2-k$ even positions after you. Again there are $n / 2$ occupied positions at saturation. In the odd case, you are better off choosing an odd position as in that case the saturated configuration consists of all of the odd positions and contains $(n+1) / 2$ persons versus $(n-1) / 2$ persons in case you choose an even position. 
In either case, choosing any odd position yields $\lceil n / 2\rceil$ persons in the saturated configuration. At this point, if the remaining positions are filled randomly, there is a distinct advantage to picking one of the positions at the end. Under the uniform random choice filling strategy, the number of persons that enter before picking a position beside you follows a negative hypergeometric distribution with parameters $N=n-\lceil n / 2\rceil$ and $a$ where $a$ is the number of positions available beside you, i.e., $a=1$ if you occupy position 1 or $n$ and $a=2$ otherwise. The expectation of such a random variable is given by $(N+1) /(a+1)$ so we get the expected time until your privacy is violated is

$$
\lceil n / 2\rceil+\frac{\lfloor n / 2\rfloor+1}{2}
$$

if you choose positions 1 or $n$ and

$$
\lceil n / 2\rceil+\frac{\lfloor n / 2\rfloor+1}{3}
$$

otherwise. Clearly, choosing an end position is to your advantage for all $n$.

\subsection{Cooperative behavior}

In this model, all persons cooperate to ensure that the configuration becomes saturated at the last possible moment, by each choosing a position that guarantees the maximum number of persons have full privacy for as long as possible. It is easy to establish that this case is very similar to the above in that as long as you choose an odd position the saturated configuration will contain $\lceil n / 2\rceil$ persons and the best overall choice turns out to be either end.

\subsection{Maximize your distance behavior}

In this model we assume that private positions will be filled in an order which maximizes the distance to any of the currently occupied positions. If more than one such position exists then a random one among them is chosen. If no position with privacy exists then a random position is chosen.

First we observe that by symmetry the number and positions of the persons when saturation is first reached are independent of the random choices made among equidistant positions and only depends on the choice that you make as the first person. Let $A(n, i)$ be number of persons in the saturated state if the first person chooses position $i, i=1, \ldots, n$. Let $B(n)$ be the number of persons in such a saturated state assuming positions 1 and $n$ are filled (not including the persons at positions 1 and $n$ ).

Assume $n>3$. (The cases $1 \leq n \leq 3$ are straightforward to analyze.) Observe that if the first person chooses position 1 (respectively, $n$ ) then the second one will choose $n$ (respectively, 1 ) and the saturated configuration will contain $2+B(n)$ persons. If the first person chooses 2 (respectively, $n-1)$ the second will choose $n$ (respectively, 1). If the first person chooses some position $i$ between 3 and $n-2$ then the second person will choose the further of 1 and $n$ 
and eventually someone will choose the other. This yields the following equation for $A(n, i)$ :

$$
A(n, i)= \begin{cases}2+B(n) & i=1, n \\ 2+B(n-1) & i=2, n-1 \\ 3+B(i)+B(n-i+1) & 3 \leq i \leq n-2 .\end{cases}
$$

Further observe that in any gap of $k>2$ unoccupied positions with the positions at either end occupied, the first of the unoccupied positions to be occupied will be the middle one (or one of the two middle ones if $k$ is even). This yields:

$$
B(n)= \begin{cases}0 & n \leq 4 \\ 1+B\left(\left\lfloor\frac{n+1}{2}\right\rfloor\right)+B\left(\left\lceil\frac{n+1}{2}\right\rceil\right) & n>4 .\end{cases}
$$

It is relatively straightforward to establish the following closed form solution for $B(n)$ :

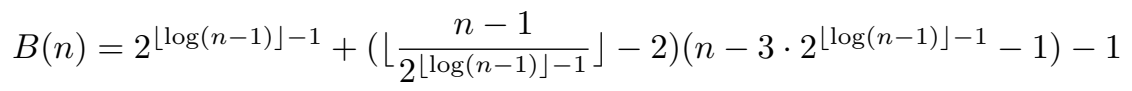

which in turn allows us to compute $A(n, i)$ for any $n$ and $i$. Unfortunately, we were not able to establish a closed form expression for $\max _{i} A(n, i)$ or show that it can be computed any faster than evaluating $A(n, i)$ for all $i$. Note that this is a pseudo-polynomial time algorithm as the input to our problem is just $n$. (See the discussion below concerning different initial configurations for more on the analysis of this algorithm.) While the function is reasonably well-behaved (piece-wise linear) it does vary widely for some values of $n$ between the extremes of $\lceil n / 3\rceil$ and $\lceil n / 2\rceil$.

If one was just concerned about maximizing the time until saturation occurs it turns out that end positions are not optimal. In fact, for some values of $n$ position 1 is the worst choice in this regard. For example, for $n$ of the form $3 \cdot 2^{k}+1$ for some $k>0, A(n, 1)=\lceil n / 3\rceil$ while $A(n,\lceil n / 3\rceil)=\lceil n / 2\rceil$.

Fortunately, one can show that once again the end positions have an advantage when we assume that the remaining positions are filled in random order. It is fairly easy to show that $A(n, 1) \geq\lceil n / 3\rceil+1$ for all $n$ not of the form $3 \cdot 2^{k}+1$ and that $A(n, i) \leq\lceil n / 2\rceil$ (at most one of every pair may be occupied) for all $n$ and $i$ and since

$$
\lceil n / 3\rceil+1+\frac{n-\lceil n / 3\rceil}{2} \geq\lceil n / 2\rceil+\frac{\lfloor n / 2\rfloor+1}{3}
$$

we see that position number 1 (and $n$ ) is always an optimal choice. (It can be verified directly that for $n$ of the form $3 \cdot 2^{k}+1$ the advantage of positions 1 and $n$ is at least $1 / 6$.) 


\subsection{Random behavior}

In this model we assume that when a person enters if the configuration is not saturated he chooses uniformly at random among all positions that provide him with privacy on both sides and that once saturation is reached he chooses uniformly among all available positions. If the first person chooses randomly like everyone else then the question of how many persons there are at the time of saturation corresponds to the unfriendly seating arrangement problem discussed above. In our case we are interested in computing whether it makes a difference which position you choose as the first person. And indeed it does. Consider the case $n=5$. If position 1 is chosen it is easy to calculate that the expected number of persons at saturation is 2.67 but if you choose position 3 you are guaranteed 3 persons at saturation so it would appear that position 3 is better. But as above it is during the filling phase that the advantage of an end takes over. In this example, position 1 expects 4.33 persons (including himself) before his privacy is violated versus the situation for position 3 where the fourth person will certainly violate his privacy.

Let $E(n, i)$ be the expected number of persons entering before your privacy is violated assuming you choose position $i$. Let $p_{i, k}$ be the probability that if you choose position $i$ the resulting saturated configuration has $k$ persons in it. Let $q_{j, k, \ell}$ be the probability that, if there are $k$ spots available after saturation and $\ell=1,2$ depending on whether you are at an end or not (indicating the number of positions you have available beside you), your privacy is violated by the $j$ th person $(j=1, \ldots, n-k)$ entering after saturation. As above we note that a saturated configuration has between $\lceil n / 3\rceil$ and $\lceil n / 2\rceil$ positions taken and so we can calculate:

$$
\begin{aligned}
E(n, i) & =\sum_{s=\lceil n / 3\rceil+1}^{n} s \sum_{j+k=s, j>0,\lceil n / 3\rceil \leq k \leq\lceil n / 2\rceil} q_{j, n-k, \ell} p_{i, k} \\
& =\sum_{s=\lceil n / 3\rceil+1}^{n} \sum_{k=\lceil n / 3\rceil, s-k>0}^{\lceil n / 2\rceil} s \cdot q_{s-k, n-k, \ell} p_{i, k} \\
& =\sum_{k=\lceil n / 3\rceil}^{\lceil n / 2\rceil} \sum_{k=\lceil n / 3\rceil+1, s-k>0}^{n}(k+s-k) \cdot q_{s-k, n-k, \ell} p_{i, k} \\
& =\sum_{k=\lceil n / 3\rceil}^{\lceil n / 2\rceil}\left(k \sum_{j=1}^{n-k} q_{j, n-k, \ell}+\sum_{j=1}^{n-k} j \cdot q_{j, n-k, \ell}\right) p_{i, k} \\
& =\sum_{k=\lceil n / 3\rceil}^{\lceil n / 2\rceil}\left(k+\sum_{j=1}^{n-k} j \cdot q_{j, n-k, \ell}\right) p_{i, k} .
\end{aligned}
$$

Using the fact that that $q_{j, k, \ell}$ is negative hypergeometrically distributed we see 
that

$$
\sum_{j=1}^{n-k} j \cdot q_{j, n-k, \ell}= \begin{cases}\frac{n-k+1}{2} & \ell=1 \\ \frac{n-k+1}{3} & \ell=2\end{cases}
$$

and so:

$$
E(n, i)= \begin{cases}\frac{n+1}{2}+(1 / 2) \sum_{k=\lceil n / 3\rceil}^{\lceil n / 2\rceil} k p_{i, k} & i=1, n \\ \frac{n+1}{3}+(2 / 3) \sum_{k=\lceil n / 3\rceil}^{\lceil n / 2\rceil} k p_{i, k} & i=2, \ldots, n-1 .\end{cases}
$$

Let $F(n, i)=\sum_{k=\lceil n / 3\rceil}^{\lceil n / 2\rceil} k p_{i, k}$. Note that $F(n, i)$ is the expected number of positions occupied at saturation if position $i$ is chosen first. But it is easy to see that

$$
F(n, i)=1+ \begin{cases}F(n-2) & i=1, n \\ F(n-3) & i=2, n-1 \\ F(i-2)+F(n-i-1) & n=3, \ldots, n-2\end{cases}
$$

where $F(n)=\sum_{i=0}^{n-1} \frac{(-2)^{i}(n-i)}{(i+1) !}$ is the expected number of positions at saturation if all choices are random established in $[3,7]$.

While $F(n)$ is monotonic as we saw above for the case $n=5$ it is not the case that $F(n, 1)>F(n, i)$ for all $i$. On the other hand, it is fairly straightforward to show that $F(n, 1)+3 / 2>F(n, i)$. The cases $i=2$ and $n-1$ follow from the monotonicity of $F(n)$. Note that

$$
\begin{aligned}
F(n) & =\sum_{i=0}^{n-1} \frac{(-2)^{i}(n-i)}{(i+1) !} \\
& =\sum_{i=0}^{\infty} \frac{(-2)^{i}(n-i)}{(i+1) !}-\sum_{i=n}^{\infty} \frac{(-2)^{i}(n-i)}{(i+1) !} \\
& =\sum_{i=0}^{\infty} \frac{(-2)^{i} n}{(i+1) !}-\sum_{i=0}^{\infty} \frac{(-2)^{i} i}{(i+1) !}+\sum_{i=0}^{\infty} \frac{(-2)^{n+i} i}{(n+i+1) !} \\
& =\frac{n}{2}\left(1-e^{-2}\right)+\frac{1-3 e^{-2}}{2}+\sum_{i=1}^{\infty} \frac{(-2)^{n+i} i}{(n+i+1) !} .
\end{aligned}
$$

For $n>0$ the last term is always greater than $-1 / 3$ and less than $2 / 3$. This implies (for $n>4$ and $2<i<n-1$ ):

$$
F(n, 1)=1+F(n-2)>\frac{n-2}{2}\left(1-e^{-2}\right)+\frac{1-3 e^{-2}}{2}+2 / 3
$$

and

$$
F(n, i)=1+F(i-2)+F(n-i-1)<\frac{n-3}{2}\left(1-e^{-2}\right)+1-3 e^{-2}+7 / 3 .
$$

These together imply $F(n, 1)+3 / 2>F(n, i)$.

Using the fact that $F(n, i) \leq\lceil n / 2\rceil$ for all $i$ and $n$ and substituting into the formula for $E(n, i)$ above we can conclude that once again positions 1 (and $n$ ) are the optimal choice for $n>8$. The remaining cases can be checked by hand. 


\section{Variations on our Theme}

\subsection{Different filling strategies}

The above analysis assumes once saturation is reached the remaining positions are filled uniformly at random. This may not be realistic as one could imagine other approaches.

Consider, for example, a lazy filling strategy whereby a new entrant, finding the configuration saturated, chooses the first available unoccupied position. Recall that we call a position private if the positions on either side of it (if they exist) are unoccupied. A non-end position is semi-private if only one of its neighbors is occupied. A position is non-private if it is not private or semiprivate. We say a person's behavior is private first if a person always chooses a private position if available. All of the behaviors we have been considering are private first.

It is fairly straightforward to show that for any behavior that follows a private first initial strategy and a lazy filling strategy, the optimal choice is always position $n$ as in this case all positions except $n-1$ must be used before your privacy is violated.

An interesting situation arises in the case where semi-private positions are preferred to non-private positions when filling. The expected time until your privacy is violated now depends on how many semi-private positions versus nonprivate positions exist at saturation and what types of gaps exist beside you (one or two unoccupied positions). Given this information the expected time of your privacy can be calculated using the appropriate negative hypergeometric distribution.

Consider what happens in the case of the distance maximizing behavior above if (1) in the initial phase when choosing between two equidistant alternatives one always chooses the lower numbered position (i.e., the lazy choice) and (2) after reaching saturation the persons choose randomly first among semiprivate positions and only after they are filled they choose randomly among non-private positions. In this case, the advantage held by the end positions disappears! If $n=8$ the expected time until privacy is violated for position 1 is 5 versus 6.33 if you choose position 3. If $n=21$ the expected length of your privacy for position 21 is 14.5 versus 15 if you choose position 3 . In fact, one can construct infinitely many $n$ for which position 1 (or position $n$ ) is not the best choice. ( $n=8$ is the smallest case for position 1 and $n=6$ the smallest for the last position.) But in each of these cases, we find that if the first position is not optimal, the last is, and vice versa. We conjecture that under the behavior above it will always be the case that either position 1 or position $n$ will be optimal. Further we conjecture that if instead of making a lazy choice for equidistant positions in the first phase but making a random choice, the advantage for both position 1 and position $n$ remains. If your filling strategy is to always fill a randomly chosen gap of size 2 in order to get a semi-private position (if one exists) in a lazy manner, then one can show that position 1 is no longer optimal (already it fails for $n=4$ ) but position $n$ is always optimal. 
For the random behavior and for any of the filling strategies considered above one can show that position $n$ remains optimal.

\subsection{Non-empty initial configuration}

More often than not when you enter a public ATM room in a busy area the configuration you observe is not the empty one. What should one do in this case?

A rule of thumb that follows from the discussion above is it likely the case that if one of the end positions is open and its single neighbor is unoccupied, you should choose that position. Note that this is not always the case! If we are in a situation where one of the ends is available but choosing it will force a gap of size 2 to form beside it, it may be the case that, if semi-private positions are preferred after saturation, choosing the end is not a good idea.

In general, the only way we know of to be certain you make the best choice is to compute for each position the expected length of privacy using the formulas provided above applying them to the subproblems created by the configuration. For example, if one is analyzing the case where maximizing the distance behavior is assumed, then one can compute the time to saturation by adding the times resulting from each of the gaps in the configuration using the formula $B(n)$ (or an appropriate modification in the case where one end of a gap is position 1 or $n$ ). After saturation, the filling time can be computed using the appropriate negative hypergeometric distribution. If the configuration is presented as $\{0,1\}$ vector then this results in a $O\left(n^{2}\right)$ time algorithm for finding the best position to choose. If the configuration is given as a list of positions then in general the algorithm is only pseudo-polynomial. We conjecture there is a polynomial time algorithm for each of the behaviors and filling strategies discussed above but have not been able to determine one for all cases.

\subsection{Dynamic situation}

Perhaps the least realistic assumptions we have made above are (1) the arrival rate of persons is constant and (2) the persons stay indefinitely once they arrive rather than leaving once they have completed their business. It is fairly evident that both the arrivals and service times of the persons are complex random processes. As a first attempt, the use of queueing theory to model this aspect of the problem seems warranted.

The simplest assumption in this regard would be that the arrival times form a Poisson process and the time required at a position is exponential distributed. As there are $n$ positions, this leads to what is termed an $\mathrm{M} / \mathrm{M} / \mathrm{n}$ queue in the standard queueing theory terminology $[6,9]$. Given $1 / \lambda$, the mean time between arrivals, and $1 / \mu$, the mean service time, it is straightforward to calculate such quantities as the expected number of "customers" in the system (either being serviced or waiting for a position to open up in case all are full), the probability that $k$ positions are free, etc. and use this information to help devise a strategy for picking a position. 
For example, if $\frac{\lambda}{\mu}<n / 2$ then you are in a region where one expects that saturation will not be reached during your visit (or even if it is the number of filled positions after saturation will be small.) In this case, if one assumes the maximizing distance behavior above then the advantage of the ends disappears. One should use the algorithm above to estimate the position that prolongs the time until saturation the longest. Generally, this will be somewhere in the largest gap of unoccupied positions and not necessarily at the end. (Again, if we assume a configuration is represented by a $\{0,1\}$ vector this will be a polynomial time algorithm.) On the other hand, if one assumes random behavior then for most configurations (ones with a sufficiently large gap) a search using a Python program suggests the best choice is one that leaves three unoccupied positions between you and either the beginning or the end of the largest gap. This appears to insure that the time to saturation in that gap will be maximized.

The above analysis assumes you have a good estimate of $\lambda$ and $\mu$ which seems unlikely. Without this information one can make a maximum likelihood estimate of $\frac{\lambda}{\mu}$ using the number of persons in the configuration upon your arrival. If the current configuration is not saturated you may as well assume that this is the steady-state and follow the guidelines described in the last paragraph for the case $\frac{\lambda}{\mu}<n / 2$. On the other hand if the configuration is saturated then you have no choice but to pick a semi-private or even non-private position. It might be interesting to investigate whether one such spot is better than another for increasing your privacy. This would seem to depend on having an estimate of the time remaining to service individuals currently in the configuration but perhaps not.

\subsection{Game-theoretic formulation}

Another objection to the approach taken above is that those arriving after the first arrival are limited to following a particular behavior. In reality they are also interested in maximizing their private time. How might considerations of more general strategies effect your choice? If the second person to enter is going to maximize his privacy based upon your choice how does that effect your choice as first. If $k$ persons enter simultaneously, is there a mechanism by which they might be led to choose a configuration that maximizes the average or maxmin privacy?

These questions suggest that our problem is in reality some sort of game played by multiple players in rounds and the tools of game theory might be applied to establish better strategies. We leave it up to experts in algorithmic game theory to formulate the right questions that might shed some light on this problem. We are hopeful this might lead to some interesting lines of research.

\subsection{Metric space generalizations}

Maintaining privacy on a line is just one of many situations where physical privacy might be desirable, in a place where people are entering (and leaving) over time. The unfriendly theatre seating arrangement problem described above 
is one such example (as is any situation with open seating). Another might be called the beach blanket problem: Frankie and Annette arrive at the beach early in the morning and want to decide where to place their beach blanket to allow for the most privacy throughout the day in order to engage in whatever activities they have planned for the day. Under various assumptions about the behavior of later arrivals, what place should they choose? This would correspond to the continuous version of our original problem.

A natural generalization of our problem that captures all of these might be termed the metric space privacy problem. You are given a metric space $(M, d)$, an $\epsilon$ (the radius of privacy), and a deterministic or randomized behavior describing the choices of points in the space of later arrivals. Given a configuration (a subset of points in $M$ ) choose a point that maximizes the (expected) time until your privacy radius is violated, i.e., a later arrival chooses a point within distance $\epsilon$ of your spot. In the theatre seating problem we take $M$ to be the vertices of an $m \times n$ grid with edge weights equal to 1 and $\epsilon=1$. In the beach blanket problem $M$ may be modelled by a simple polygon (perhaps even a rectangle) with the Euclidean distance measure and $\epsilon$ may depend upon how much privacy you need. In the continuous problem $M$ is a unit interval and $\epsilon$ is a function of a person's width and privacy needs.

One suspects that some instances of these problems will turn out to be $N P$ hard as it would appear that in some cases you would be forced to solve a version of the obnoxious facility location problem that in some cases is known to be $N P$-hard. Consideration of a given metric space can be combined with dynamics and/or game theory to yield more problems. We feel there is the potential for many interesting open questions in this area.

\section{Conclusions}

Our main conclusion is that when faced with the decision of what ATM to choose upon entering the room, in order to maximize your privacy, you should probably choose the one furthest from the door if it is available and the one next to it is unoccupied. For a vast majority of the (what we consider) natural behaviors this choice is optimal (in the sense defined above). Beyond this observation, we feel that this problem leads to many interesting variations that are worthy of investigating further.

\section{Acknowledgments}

We thank Peter Boothe for suggesting useful references. This research was supported in part by Natural Sciences and Engineering Research Council of Canada (NSERC) and Mathematics of Information Technology and Complex Systems (MITACS). 


\section{References}

[1] P. Flajolet, "A Seating Arrangement Problem," http://algo.inria.fr/libraries/autocomb/fatmen-html/fatmen1.html.

[2] D. Freedman and L. Shepp, "An Unfriendly Seating Arrangement Problem." SIAM Review, 4 (1962), pp. 150.

[3] H. D. Friedman and D. Rothman, "Solution to An Unfriendly Seating Arrangement Problem," SIAM Review, 6 (1964), pp. 180-182.

[4] K. Georgiou, E. Kranakis and D. Krizanc, "Random Maximal Independent Sets and the Unfriendly Theater Arrangement Problem," Discrete Mathematics, 309 (2009), pp. 5120-5129.

[5] Lance Hoffman, "Computers and Privacy: A Survey," Computing Surveys, 1 (1969), pp. 85-103.

[6] L. Kleinrock, Queueing Systems, Volume 1: Theory, Wiley, Hoboken, NJ, 1975.

[7] J. K. MacKenzie, "Sequential Filling of a Line by Intervals Placed at Random and Its Application to Linear Absorption," Journal of Chemical Physics, 37 (1962), pp. 723-728.

[8] C. Paola, "A Survey of Obnoxious Facility Location Problems," TR-99-11, University of Pisa, Dept. of Informatics, 1999.

[9] S. M. Ross, "Stochastic Processes", John Wiley \& Sons, Inc., 2nd edition, 1996.

[10] R. Vogt, M. A. Nascimento and J. Harns, "On the Tradeoff between UserLocation Privacy and Queried-Location Privacy in Wireless Sensor Networks," AdHoc Now 2009, pp. 241-254. 